\title{
On the spectral distribution of kernel matrices related to radial basis functions ${ }^{\text {th }}$
}

\author{
Andy Wathen ${ }^{\mathrm{a}, \mathrm{b}}$, Shengxin Zhu ${ }^{\mathrm{a}, \mathrm{b}}$ \\ ${ }^{a}$ Numerical Analysis Group, The University of Oxford, 24-29 St Giles, OX1 3LB, Oxford, UK \\ ${ }^{b}$ Oxford Center for Collaborative and Applied Mathematics, 24-29 St Giles, OX1, 3LB, Oxford, UK
}

\begin{abstract}
This paper focuses on the spectral distribution of kernel matrices related to radial basis functions. By relating a contemporary finite-dimensional linear algebra problem to a classical problem on infinite-dimensional linear integral operator, the paper shows how the spectral distribution of a kernel matrix relates to the smoothness of the underlying kernel function. The asymptotic behaviour of the eigenvalues of a infinite-dimensional kernel operator are studied from a perspective of low rank approximation - approximating an integral operator in terms of Fourier series or Chebyshev series truncations. Further, we study how the spectral distribution of interpolation matrices of an infinite smooth kernel with flat limit depends on the geometric property of the underlying interpolation points. In particularly, the paper discusses the analytic eigenvalue distribution of Gaussian kernels, which have important application on stable computing of Gaussian radial basis functions.
\end{abstract}

Keywords: eigenvalues, radial basis functions, spectral distribution, ill-conditioning, integral equation of the first kind

2010 MSC: 42A10, 45A25, 45B05, 45C05, 47A52, 47A75

\section{Motivation}

The increasing importance of high-dimensional problems and scattered data processing motivates us to investigate the properties of kernel matrices related to radial basis functions

\footnotetext{
«This research is supported by Award no KUK-C1-013-04, made by King Abdullah University of Science of Technology

Email addresses: wathen@maths.ox.ac.uk (Andy Wathen), shengxin.zhu@maths.ox.ac.uk (Shengxin Zhu)

Preprint submitted to Oxford preprint

May 15, 2013
} 
(RBFs). RBFs have been shown as an attractive approach for scattered data approximation [47]. They can guarantee invertible linear systems [25], have good approximating quality, and are promising to deal with high dimensional problems and complex geometry domain. These attractive properties make RBFs one of the foundations of many multivariate approximation based methods and techniques such as surface reconstruction, mesh-free methods, machine learning, global optimization, surface computing. However, research has found that linear systems related to some globally supported radial basis functions can be highly illconditioned for standard basis which can result in extremely large condition number. The condition number of a matrix $A$ is defined by $\kappa(A)=\left\|A^{-1}\right\|\|A\|$, where $\|\cdot\|$ denotes the standard 2-norm of matrices. It depends on the ratio of the largest magnitude eigenvalues to the smallest magnitude eigenvalue for symmetric matrices; many RBFs interpolation matrices are of this type.

Stable computing the ill-conditioned linear system usually need to change the standard basis to an better set of basis and to explore the spectral information. For example, Gaussian radial basis functions $e^{-\varepsilon^{2}\|\mathbf{x}\|^{2}}, \mathbf{x} \in \mathbb{R}^{d}$ with flat limit - the cases corresponding to $\varepsilon \rightarrow 0$ - can result in highly ill-conditioned linear systems, [14, 22]. Stable computing such highly illconditioned linear systems requires relevant spectral information of Gaussian kernel, see [10, $12,23]$. In the context of finite element method(FEM), based on the spectral distribution of Galerkin mass matrices, a simple and efficient diagonal preconditioner has been developed to speed up the computation [46]. Such work has already vividly shown that priori information on the spectral distribution of a linear system is useful and sometime essential to design a simple and efficient preconditioner for fast and stable solvers.

Pioneering results on the spectral information of RBFs interpolation matrices focused primarily on the estimation of the smallest (magnitude) eigenvalues of the underlying interpolation matrices. There are two fundamental motivations behind such work: first, for understanding the solvability of high-dimensional interpolation problem with certain radial basis functions, to prove the related matrices do not have any zero eigenvalue. [1,25]; second, for understanding the stability of theoretical computing, to estimate the upper bound of the norm of the inverse of an interpolation matrix, or to estimate the condition number of the 
underlying interpolation matrix, in this case, sharper estimation on the smallest magnitude eigenvalue is often required [3, 26, 28, 29, 41, 42].

Spectral distribution can further characterize the conditioning issues and reveal more information than the conditioning number does[40]. Our recent research finds that several other important computing issues are also closely related to the spectral distribution. Further understanding the spectral information of RBFs interpolation matrices is still necessary.

One main idea of the paper is to relate the RBFs interpolation scheme to the discrete integral equation of the first kind, see Section 2. Instead of considering the finite-dimensional linear algebra problem directly, we investigate the corresponding classical infinite-dimensional linear operator problem. Once put a contemporary problem in a proper perspective, powerful tools are already available and many results can be applied to explore the underlying problem. Combining several scattered results, we obtain results on how the decay of eigenvalues of kernel matrices of RBFs is closely related to the smoothness of the underlying RBFs (Theorem 4 and Theorem 5). The results perhaps are not striking new, but we have not seen the results elsewhere. Several established results - Theorem 1, Theorem 3 and Theorem 6 - are well known in the community of linear integral equations, but it seems that these results drew little attention from the community of RBFs. The difference and connections between these theorems are discussed.

The remaining of the paper is organised as follow. Section 2 introduce the connection between the RBFs interpolation problem and the Fredholm integral equation of the first kind. Also introduced are several relevant lemmas including Weyl-Courant minimax principle. Main results are discussed in Section 3. Finally, we discuss other relevant results in existence, the connection with RBF-QR method, and possible applications.

\section{Preliminaries}

Consider the following integral equation of the first kind

$$
\int_{\Omega} K(\mathbf{x}, \mathbf{y}) \alpha(\mathbf{y}) d \mathbf{y}=f(\mathbf{x})
$$


where $\mathbf{x}, \mathbf{y} \in \mathbb{R}^{d}$. We call $K(\mathbf{x}, \mathbf{y})$ a kernel function in $\mathbb{R}^{d}$. Further, if $K(\mathbf{x}, \mathbf{y})$ satisfies $\int_{\Omega} K(\mathbf{x}, \mathbf{y}) \alpha(\mathbf{y}) \alpha(\mathbf{x}) d \mathbf{x} d \mathbf{y}>0$ for any non-zero function $\alpha(\mathbf{x})$, then $K(\mathbf{x}, \mathbf{y})$ is positive definite. If a simple quadrature rule is applied to sample $\alpha(\mathbf{y})$, then we get a discrete equation

$$
\sum_{j=1}^{N} K\left(\mathbf{x}, \mathbf{y}_{j}\right) w_{j} \alpha\left(\mathbf{y}_{j}\right)=f(\mathbf{x})
$$

Further collocating at $\mathbf{x}_{1}, \mathbf{x}_{2}, \ldots, \mathbf{x}_{N}$ gives

$$
\sum_{j=1}^{N} K\left(\mathbf{x}_{i}, \mathbf{y}_{j}\right) w_{j} \alpha\left(\mathbf{y}_{j}\right)=f_{i}, i=1,2, \ldots, N
$$

where $f_{i}=f\left(\mathbf{x}_{i}\right)$. Let $K(\mathbf{x}, \mathbf{y})=\phi(\|\mathbf{x}-\mathbf{y}\|)$, then we can write these equations as the linear system

$$
\left(\begin{array}{cccc}
\phi\left(\left\|\mathbf{x}_{1}-\mathbf{y}_{1}\right\|\right) & \phi\left(\left\|\mathbf{x}_{1}-\mathbf{y}_{2}\right\|\right) & \cdots & \phi\left(\left\|\mathbf{x}_{1}-\mathbf{y}_{N}\right\|\right) \\
\phi\left(\left\|\mathbf{x}_{2}-\mathbf{y}_{1}\right\|\right) & \phi\left(\left\|\mathbf{x}_{2}-\mathbf{y}_{2}\right\|\right) & \cdots & \phi\left(\left\|\mathbf{x}_{2}-\mathbf{y}_{N}\right\|\right) \\
\vdots & \vdots & \ddots & \vdots \\
\phi\left(\left\|\mathbf{x}_{M}-\mathbf{y}_{1}\right\|\right) & \phi\left(\left\|\mathbf{x}_{M}-\mathbf{y}_{2}\right\|\right) & \cdots & \phi\left(\left\|\mathbf{x}_{M}-\mathbf{y}_{N}\right\|\right)
\end{array}\right)\left(\begin{array}{c}
\alpha_{1} \\
\alpha_{2} \\
\vdots \\
\alpha_{N}
\end{array}\right)=\left(\begin{array}{c}
f_{1} \\
f_{2} \\
\vdots \\
f_{M}
\end{array}\right)
$$

where $\alpha_{j}=w_{j} \alpha\left(\mathbf{y}_{j}\right), j=1,2, \ldots, N$. We denote the matrix in (4) by $A_{\phi, \mathcal{X}}$, where $\mathcal{X}$ denotes the set $\left\{\mathbf{x}_{1}, \mathbf{x}_{2}, \ldots, \mathbf{x}_{N}\right\}$. When $\mathbf{x}_{i}=\mathbf{y}_{i}, 1 \leq i \leq N$, the linear system (4) is the same as that obtained for interpolation matrices with the radial basis function $\phi$ at the points $\mathbf{x}_{1}, \mathbf{x}_{2}, \ldots, \mathbf{x}_{N}$. In this paper we call the matrix in (4) a kernel matrix.

On the other hand, consider the interpolation scheme with a sum of translates of a radial basis function

$$
s(\mathbf{x})=\sum_{j=1}^{N} \alpha_{j} \phi\left(\left\|\mathbf{x}-\mathbf{y}_{j}\right\|\right) .
$$

If $s(\mathbf{x})$ interpolates an unknown function, $f(\mathbf{x})$, on the data set $\mathcal{X}$, and further $\mathbf{x}_{j}=\mathbf{y}_{j}$, $1 \leq j \leq N$, it results in the same linear system (4). In this case we call the matrix $A_{\phi, \mathcal{X}}$ an interpolation matrix. In this paper, we use kernel matrices for short.

We investigate the spectral distribution of kernel matrices related to radial basis function 
by studying the infinite dimensional linear operator

$$
\mathcal{K} \Psi(\mathbf{x})=\int_{\Omega} K(\mathbf{x}, \mathbf{y}) \Psi(\mathbf{y}) d \mathbf{y}
$$

where $K(\mathbf{x}, \mathbf{y})=\phi(\|\mathbf{x}-\mathbf{y}\|)$ is a radial function $\phi$. To investigate the spectral distribution of the operator $\mathcal{K}$ in (6), consider the following well known Weyl-Courant minimax principle.

Lemma 1 (The Weyl-Courant minimax principle). Let $\mathcal{K}$ be a compact symmetric operator on a Hilbert space $\mathcal{H}$ with eigenvalues

$$
\left|\lambda_{0}\right| \geq\left|\lambda_{1}\right| \geq\left|\lambda_{2}\right| \geq \cdots \geq\left|\lambda_{n}\right| \geq \cdots
$$

and $\mathcal{S}$ be any operator of rank $\leq n$, then $\|\mathcal{K}-\mathcal{S}\| \geq\left|\lambda_{n}\right|$.

The reader is directed to [24] for proof of the lemma. In [24], Little and Reade apply the Weyl-Courant minimax principle to tails of the Chebyshev expansion for analytic kernels, and conclude that the eigenvalues of an analytic kernel on a finite interval go to zeros at least as fast as $R^{-n}$ for some fixed $R>1$. The proof depends on the estimation of Chebyshev coefficients of analytic functions. Some results used in their proof in fact can date back to Bernstein [4] and relate to the so-called Bernstein's ellipse, denoted as $\mathcal{E}_{\rho}$ [45, p.56]. The ellipse, $\mathcal{E}_{\rho}$, has foci at \pm 1 , and the sum of the semi-axes equals $\rho>1$.

Lemma 2 ([4][45, p.57]). Let a function, $f$, analytic in $[-1,1]$ be analytically continuable to the open Bernstein ellipse $\mathcal{E}_{\rho}$, where it satisfies $|f(z)| \leq M$ for some $M$. Then its Chebyshev coefficients, $a_{n}$, satisfy $a_{0} \leq M$ and

$$
\left|a_{n}\right| \leq 2 M \rho^{-n}, n \geq 1
$$

Its Chebyshev truncations satisfy

$$
\left\|f-S_{n}\right\| \leq \frac{2 M \rho^{-n}}{\rho-1}
$$


and its Chebyshev interpolants satisfy

$$
\left\|f-p_{n}\right\| \leq \frac{4 M \rho^{-n}}{\rho-1}
$$

where $S_{n}(x)=\sum_{k=0}^{n} a_{k} T_{k}(x)$, and $p_{n}(x)$ is the polynomial obtained by interpolation in Chebyshev points.

The formula (7) is due to Bernstein, and the second part of the lemma can be found in $[45$, p.57, thm. 8.2].

A function of bounded variation on $\mathbb{R}, f(x)$, is a real-valued function, it is an integrable function, say, $f(x) \in L(\mathbb{R})$, and, $\mathrm{V}$, the supremum of $\int f(x) d g(x)$ over all $g(x) \in C^{1}(\mathbb{R})$ with $|g(x)|<1$ is finite. If $f(x)$ is continuous, then the supremum of $\sum_{j=1}^{N}\left|f\left(x_{j}\right)-f\left(x_{j-1}\right)\right|$ is bounded over all finite samples $x_{0}, x_{1}, \ldots, x_{N}$. For finitely differentiable functions with the highest derivatives of bounded variations, we consider the following result.

Lemma 3 ([45, p.52-p.53]). For any integer $\nu \geq 0$, let $u$ and its derivatives $u^{\prime}, \ldots, u^{(\nu-1)}$ be absolutely continuous on $[-1,1]$, and $u^{(\nu)}$ be of bounded variation $V$, then for any $n \geq \nu+1$, the Chebyshev coefficients of u satisfy

$$
\left|a_{n}\right| \leq \frac{2 V}{\pi(n-\nu)^{\nu+1}}, n>\nu
$$

Its Chebyshev truncations $S_{n}$ satisfy

$$
\left\|u-S_{n}\right\| \leq \frac{2 V}{\pi \nu(n-\nu)^{\nu}}, n>\nu
$$

and its Chebyshev interpolants satisfy

$$
\left\|f-p_{n}\right\| \leq \frac{4 V}{\pi \nu(n-\nu)^{\nu}}, n>\nu
$$

For details of the proof of Lemma 3, the reader is directed to Trefethen's new book [45, thm 7.1 and thm 7.2]. 
Besides Chebyshev truncations, we also consider the Fourier series truncations. Such an approximation is valid for a kernel function which is Lebesgue integrable, not necessarily analytic, and it can be generalized to higher dimensional space. Here, we demonstrate the idea with the simplest one dimensional case. For a Lebesgue integrable function $u$ on $\Omega$, say, $u \in L^{1}(\Omega)$, the $n$-th Fourier coefficient of $u$ is defined by

$$
\hat{u}_{n}:=\hat{u}(n)=\frac{1}{T} \int_{-T / 2}^{T / 2} u(t) e^{-i n t} d t
$$

for a $T$-periodic function, $u$, on $\Omega=[-T / 2, T / 2]$, where $T$ could be finite or infinite. If there is no confusion we use $\hat{u}_{n}$ for short, otherwise we use $\hat{u}(n)$. The Fourier series $S[u]$ of a function $u \in L^{1}(\Omega)$ is the trigonometric series

$$
S[u] \sim \sum_{n=-\infty}^{\infty} \hat{u}_{n} e^{i n t}
$$

The $n$-th Fourier series truncation is denoted as $S_{n}[u]=\sum_{k=-n}^{n} \hat{u}_{n} e^{i n t}$. Remembering the Euler's formula $e^{i t}=\cos t+i \sin t$, the Fourier series of a function $u \in L^{1}(\Omega)$ is equal to

$$
\frac{A_{0}}{2}+\sum_{n=1}^{\infty}\left(A_{n} \cos n t+B_{n} \sin n t\right)=\frac{A_{0}}{2}+\sum_{n=1}^{\infty} \sqrt{A_{n}^{2}+B_{n}^{2}} \sin \left(n t+\theta_{n}\right)
$$

where $A_{n}=\hat{u}_{n}+\hat{u}_{-n}, B_{n}=i\left\{\hat{u}_{n}-\hat{u}_{-n}\right\}$ and $\arcsin \theta_{n}=B_{n} / \sqrt{A_{n}^{2}+B_{n}^{2}}$. In particularly, when $u$ is an even function, $\hat{u}_{n}=\hat{u}_{-n}$,

$$
S_{n}=\hat{u}(0)+2 \sum_{k=1}^{n} \hat{u}_{n} \cos n t
$$

As discussed above, it is clear that the Fourier series truncation $S_{n}$ is a function of finite $\operatorname{rank} n+1$.

According to standard classical Fourier analysis, Fourier coefficients have the following properties, for example, see [20, p.3].

Proposition 1. If $u \in L^{1}(\Omega)$, then 
(a) denote $u_{\tau}=u(t-\tau), \tau \in \Omega$; then $\hat{u}_{\tau}(n)=\hat{u}_{n} e^{-i n \tau}$;

(b) $\left|\hat{u}_{n}\right| \leq \frac{1}{T} \int|u(t)| d t=\|u\|_{L^{1}}$;

(c) denote $u_{\varepsilon}(t)=u(\varepsilon t)$ for some $\varepsilon>0$, then $\hat{u}_{\varepsilon}(n)=\frac{1}{\varepsilon} \hat{u}\left(\frac{n}{\varepsilon}\right) ;{ }^{1}$

(d) if $u$ has $\nu$-the order derivative, then $\hat{u}^{(\nu)}(n)=(\text { in })^{\nu} \hat{u}(n)$;

(e) (The Riemann-Lebesgue Lemma) $\lim _{|n| \rightarrow \infty} \hat{u}(n)=0$.

If $u$ is a $\nu$-times differentiable function and $u^{(\nu)} \in L^{1}(\Omega)$, then according to the RiemannLebesgue Lemma

$$
\lim _{|n| \rightarrow \infty} \hat{u}^{(\nu)}(n)=\lim _{|n| \rightarrow \infty}(i n)^{\nu} \hat{u}(n)=0,
$$

which suggest $\hat{u}(n)=o\left(\frac{1}{n^{\nu}}\right)$ as $|n| \rightarrow \infty$. The small " $o$ " symbol is defined in the standard way : $u(k)=o(g(k))$ as $k \rightarrow \infty$ if $\lim _{k \rightarrow \infty}|u(k)| /|g(k)|=0$.

If $u$ is of bound variation $V$ on $\Omega$, then integrate (13) by parts

$$
|\hat{u}(n)|=\left|\frac{1}{T} \int e^{-i n t} u(t) d t\right|=\left|\frac{1}{i T n} \int u(t) d e^{-i n t}\right| \leq \frac{2 V}{T|n|} .
$$

In this case $\hat{u}(n)=\mathcal{O}\left(\frac{1}{|n|}\right)$, i.e. as $|n| \rightarrow \infty$, there exist a constant $C$ such that $|\hat{u}(n)|<C /|n|$. Similarly if $u$ has up to $\nu$-1-time continuous derivatives and the $\nu$-th derivative is of bounded variation and belongs to $L^{1}(\Omega)$, then $\hat{u}(n)=\mathcal{O}\left(1 / n^{\nu+1}\right)$.

One case of great interest is the square integrable function $u$ on $\Omega$, say, $u \in L^{2}(\Omega)$. In this case, $u=\lim _{n \rightarrow \infty} \sum_{k=-n}^{n} \hat{u} e^{i k t}$, in the $L^{2}$ norm. And further we have

$$
\sum_{n=-\infty}^{\infty}\left|\hat{u}(n)^{2}\right|=\frac{1}{T} \int|u(t)|^{2} d t
$$

Because the series in (19) is convergent, further suppose $\hat{u}(n)>0$, then $\hat{u}(n)^{2}=o(1 / n)$ and $\hat{u}(n)=o\left(1 / n^{1 / 2}\right)$. Similarly, if $u$ is $k$-times differentiable, and $u^{(k)} \in L^{2}(\Omega)$, then $\hat{u}^{(k)}(n)=o\left(1 / n^{k+1 / 2}\right)$. By such standard analysis, see [20] for example, we have

\footnotetext{
${ }^{1} \hat{u}\left(\frac{n}{\epsilon}\right)$ is defined similarly as the Fourier coefficients, but note that $\frac{n}{\varepsilon}$ may not be an integer.
} 
Lemma 4 (Fourier coefficients of differentiable functions). Let $u$ be a square integral function on $\Omega$ with Fourier transform $\hat{u}$ and Fourier coefficients $\hat{u}_{n}$.

1. If $u$ has $\nu-1$ continuous derivatives in $L^{2}(\Omega)$ for some $\nu \geq 0$, and the $\nu^{\text {th }}$ derivative is of bounded variation, then $\hat{u}_{n}=\mathcal{O}\left(|n|^{-\nu-1}\right)$ as $|n| \rightarrow \infty$.

2. If $u \in L^{2}(\Omega)$ and $u \in C^{\infty}$, then $\hat{u}_{n}=\mathcal{O}\left(|n|^{-\nu}\right)$ as $|n| \rightarrow \infty$ for every $\nu \geq 0$.

3. If $u$ has up to $\nu$ times continuous derivatives and $u^{(\nu)} \in L^{2}(\Omega)$, then $\hat{u}(n)=o\left(n^{-\nu-1 / 2}\right)$.

\section{Main results}

The eigenvalue problem of the linear integral operator (6) has been well-studied for many years since the work of Fredholm in 1903 [16]. There are many insightful results in the literature, see $[8,9,16,18,21,24,31-39,44,48]$ for example. This paper only collects the most relevant and brief results, provides alternative simple proofs, and develops new results.

Theorem 1 (Weyl[48, p.449-450]). If the kernel function $K(x, y)=K(y, x)$ and $\frac{\partial K^{\nu}(x, y)}{\partial^{\nu} x}$ exists and is continuous, then the magnitude of its eigenvalues decays approximately at the order $\left|\lambda_{n}\right|=o\left(n^{-\nu-1 / 2}\right)$.

The original result of Weyl only states this results for the case $\nu=1$, and states it as $\lim _{n \rightarrow \infty} n^{3 / 2} \lambda_{n}=0[48, \mathrm{p} .449]$.

If the kernel function is positive definite, sharper results hold [7, 17, 37].

Theorem 2 (Reade-Ha). If a kernel function $K(x, y)$ is positive definite, $2 \pi$-periodic in $x$ and $y$, and $\nu$ times continuously differentiable, then

1. $\lambda_{n}=\mathcal{O}\left(n^{-\nu-1}\right)$;

2. for even $\nu$, the shaper result $\sum_{n=1}^{\infty} n^{\nu} \lambda_{n}<\infty$ holds.

Remark 1. As far as we know, the above theorem was first proved for $\nu=1$ in [33] and then for the general case [34]. The second part of the theorem was initially believed to be true for all $\nu$ [17], but Reade [37] constructs a counter example showing that the second part doesn't hold for odd $p$. The eigenvalues of positive kernels have also been considered in [5, 6, 11] with additional constraints. 
It has been noted that both Theorem 1 and Theorem 2 are sharp [36].

Without the positive definite constraint, similar results can be obtained according to the following results which state the essential connection between the Fourier coefficients of a $2 \pi$-periodic kernel function and the eigenvalues of its corresponding integral operator.

Theorem 3 (Hille-Tamarkin[18, p.10]). If $K(x, y)=k(x-y)$ and $k(x) \in L^{2}[-\pi, \pi]$ is a periodic kernel, then $\lambda_{n}=2 \pi \hat{k}_{n}$, where $\hat{k}_{n}=\frac{1}{2 \pi} \int_{-\pi}^{\pi} k(x) e^{i n x} d x$ is the Fourier coefficient of the kernel function.

Applying Lemma 4 and Theorem 3, it is easy to show the following results.

Theorem 4. Let $K(x, y)=k(x-y)$, where $k(x)$ is a $2 \pi$ periodic and square integrable function on $[-\pi, \pi]$. Further let $\left|\lambda_{n}\right|$ be the $n^{\text {th }}$ largest eigenvalue in magnitude of its corresponding integral operator, then

1. if $k(x) \in C^{\nu}$, for some natural number $\nu>0$ and its $\nu$-th derivative is of bounded variation, then $\left|\lambda_{n}\right|=\mathcal{O}\left(n^{-\nu-1}\right)$ as $n \rightarrow \infty$;

2. if $k(x) \in C^{\infty}$, then $\left|\lambda_{n}\right|=\mathcal{O}\left(n^{-\nu}\right)$ as $n \rightarrow 0$ for any $\nu>0$;

3. if $k(x)$ has up to $\nu$ times continuous derivatives and the $\nu$-th derivative is square integrable, then $\left|\lambda_{n}\right|=o\left(n^{-\nu-1 / 2}\right)$.

Now, we consider methods for more general cases.

\subsection{First method: truncated Fourier series Approximation}

For simplicity, we consider the compactly supported radial basis functions; such functions are even, though themselves are not necessarily periodic but can be extended to be periodic on the real line. Let $k(x)$ be a square integrable even function in $\mathbb{R}$, Its Fourier series truncation has the form in (16). Furthermore,

$$
\lim _{n \rightarrow \infty}\left\|k(x)-S_{n}\right\|_{L^{2}}=0 .
$$


This implies $\sum_{n}^{\infty}|\hat{k}(n)|^{2}$ is convergent. According to the translation property of Fourier coefficient, Proposition 1(a),

$$
k(x-y) \sim \hat{k}(0)+\sum_{n=1}^{\infty} \hat{k}(n) e^{-i n y} \cos n x
$$

since $\left|\hat{k}_{y}(n)\right|^{2}=\left|\hat{k}(n) e^{-i n y}\right|^{2}=|\hat{k}(n)|^{2}$, and thus

$$
\left\|k(x-y)-S_{n}\left[k_{y}\right]\right\|_{L^{2}}^{2}=\sum_{m=n+1}^{\infty}\left|\hat{k}_{y}(m)\right|^{2}=\sum_{m=n+1}^{\infty}|\hat{k}(m)|^{2}
$$

According to the Weyl-Courant minimax principle, the $n+1$-th eigenvalue of the kernel function $k(x, y)$ satisfies

$$
\left|\lambda_{n+1}\right|^{2} \leq\left\|k(x-y)-S_{n}\left[k_{y}\right]\right\|_{L^{2}}^{2}=\sum_{m=n+1}^{\infty}|\hat{k}(m)|^{2}
$$

Suppose $k(x)$ satisfies Lemma 4.1, then we get

$$
\left|\lambda_{n+1}\right|^{2} \leq \sum_{m=n+1}^{\infty}|\hat{k}(m)|^{2} \leq \sum_{m=n+1}^{\infty}\left|C m^{-2 \nu-2}\right| \leq \int_{n+1}^{\infty} \frac{C}{t^{2 \nu+2}} d t=\frac{C}{(2 \nu+1)} \frac{1}{(n+1)^{2 \nu+1}}
$$

Thus, $\left|\lambda_{n}\right|=\mathcal{O}\left(n^{-\nu-1 / 2}\right)$. Similarly for $k^{(\nu)} \in L^{2}(\Omega)$, we have $\left|\lambda_{n}\right|=o\left(n^{-\nu}\right)$.

Theorem 5. Let $\phi$ be a radial basis function in $L^{2}(\Omega)$, then

1. if $\phi$ has $\nu-1$ continuous derivatives for some $\nu>0$ and the $\nu^{\text {th }}$ derivative is of bounded variation, then its eigenvalues decay at least in the order $\mathcal{O}\left(n^{-\nu-1 / 2}\right)$.

2. if $\phi$ has $\nu-1$ continuous derivatives and $\phi^{(\nu)} \in L^{2}(\Omega)$, then the eigenvalues of the corresponding linear operator decay in the order $o\left(n^{-\nu}\right)$.

With Proposition 1.(c) and a few additional computations, we obtain the following results for scaled radial basis functions.

Corollary 1. Let $\phi_{\epsilon}(x)=\phi(\epsilon x), \epsilon>0$ be a scaled radial basis function in $L^{2}(\Omega)$, then 
1. if $\phi$ has $\nu-1$ continuous derivatives for some $\nu>0$ and the $\nu^{\text {th }}$ derivative is of bounded variation, then its eigenvalue decay (4) decay at least in the order $\mathcal{O}\left(\left(\frac{\epsilon}{n}\right)^{-\nu-1 / 2}\right)$.

2. $\phi$ has $\nu$ continuous derivatives and $\phi^{(\nu)} \in L^{2}(\Omega)$, then the eigenvalues of the corresponding linear operator decay in the order $o\left(\left(\frac{\epsilon}{n}\right)^{-\nu}\right)$.

The following method employing Chebyshev truncation brings sharper results.

\subsection{Second method: truncated Chebyshev series approximation}

Using truncated Chebyshev series approximations to an analytic function has been studied in [24]. As mentioned, two key points in their poof are the Weyl-Courant minimax principle and Bernsten's results (7). A slightly different formula to (8) is needed. The Chebyshev expansion of a kernel function is

$$
K(x, y)=\frac{1}{2} a_{0}(y)+\sum_{\ell=1}^{\infty} a_{\ell}(y) T_{\ell}(x)
$$

Thus the estimation of $a_{\ell}(y)$ is needed. For details see [24].

Theorem 6 (Little-Reade [24]). If $K(x, y)=K(y, x) \in C[-1,1]^{2}$, and for each $t \in[-1,1]$ there is an analytic continuation to $K(z, y)$ for $z$ inside the ellipse $\mathcal{E}_{\rho}$, which is uniformly bounded in $z, y$ in this range, then the eigenvalues corresponding to the kernel function $K(x, y)$ decay in the order $\left|\lambda_{n+1}\right|=\mathcal{O}\left(\rho^{-n}\right)$.

Proof. See [24], or one can directly apply Lemma 2.

Now consider the scaled kernel function $K(\epsilon x, \epsilon y)$ in the unit square,

$$
\mathcal{K} f(\epsilon x)=\int_{|\epsilon t| \leq 1} K(\epsilon x, \epsilon t) f(\epsilon t) d(\epsilon t) .
$$

Then the operator (26) is equivalent to the following

$$
\mathcal{K} f(X)=\int_{-1 / \epsilon}^{1 / \epsilon} K(X, T) f(T) d(T) .
$$


Suppose $\mathcal{E}_{\rho_{\epsilon}}$ denotes the ellipse with foci at $\pm \frac{1}{\epsilon}$ and semi-axis sum $\rho_{\epsilon}$, then $\rho_{\epsilon}=\frac{\rho}{\epsilon}$. If $\epsilon<1$ and the kernel function $K(x, y)$ is analytic in $\mathcal{E}_{\rho_{\epsilon}}$, we have the shaper result.

Theorem 7. The scaled eigenvalues of the scaled kernel $K(\epsilon x, \epsilon y), \epsilon<1$, decay in the order $\left|\lambda_{n+1}\right|=\mathcal{O}\left(\rho_{\epsilon}^{-n}\right)=\mathcal{O}\left(\epsilon^{n} \rho^{-n}\right)$.

When $\epsilon \geq 1$, the factor $\epsilon^{n}$ does not work, because the ellipse $\mathcal{E}_{\rho_{\epsilon}} \subset \mathcal{E}_{\rho}$ which can not give a tighter estimation on $a_{k}$ in (7). It is observed that the case $\epsilon \rightarrow 0$ corresponds to the basis functions tending to flatness; the smallest eigenvalue of the kernel matrix will become smaller.

Similarly, Lemma 3 can be used to obtain similar results as Theorem 5 according to Chebyshev series truncation. The proof involves additional complex analysis results, the reader is directed to $[24,45]$ for details.

\subsection{Separable Kernels in $\mathbb{R}^{d}$}

A separable kernel in $\mathbb{R}^{d}$ can be expressed as the product of multiple kernels, say

$$
K(\mathbf{x}, \mathbf{y})=K_{1}\left(x_{1}, y_{1}\right) K_{2}\left(x_{2}, y_{2}\right) \cdots K_{d}\left(x_{d}, y_{d}\right), \mathbf{x}, \mathbf{y} \in \mathbb{R}^{d}, x_{i}, y_{i} \in \mathbb{R}
$$

Such kernel exists and has been considered for long time, see [44] for example. The famous example is the Gaussian radial basis functions $\exp \left(-\|\mathbf{x}-\mathbf{y}\|^{2}\right)$.

Consider the simplest separable kernel in the case $d=2$, if there exist an analytical eigenfunction expansions for each $K_{i}, i=1,2$,

$$
K_{1}\left(x_{1}, y_{1}\right)=\sum_{m=1}^{\infty} \lambda_{m} \varphi_{m}\left(x_{1}\right) \varphi_{m}^{*}\left(y_{1}\right) \text { and } K_{2}\left(x_{2}, y_{2}\right)=\sum_{n=1}^{\infty} \mu_{n} \psi_{n}\left(x_{2}\right) \psi_{n}^{*}\left(y_{2}\right)
$$

and further $\left\{\lambda_{k}\right\}_{k=1}^{\infty}$ and $\left\{\mu_{k}\right\}_{k=1}^{\infty}$ are absolute convergent, then according to Mercer theorem 
$[30$, p.96]

$$
\begin{aligned}
& \iint K_{1}\left(x_{1}, y_{1}\right) K_{2}\left(x_{2}, y_{2}\right) \varphi_{p}\left(y_{1}\right) \psi_{q}\left(y_{2}\right) d y_{1} d y_{2} \\
= & \iint \varphi_{p}\left(y_{1}\right)\left(\sum_{m=1}^{\infty} \lambda_{m} \varphi_{m}\left(x_{1}\right) \varphi_{m}^{*}\left(y_{1}\right)\right) \psi_{q}\left(y_{2}\right)\left(\sum_{n=1}^{\infty} \mu_{n} \psi_{n}\left(x_{2}\right) \psi_{n}^{*}\left(y_{2}\right)\right) d y_{1} d y_{2} \\
= & \lambda_{p} \mu_{q} \varphi_{p}\left(x_{1}\right) \psi_{q}\left(x_{2}\right) .
\end{aligned}
$$

Note that when the kernel is symmetric, then $\varphi_{m}^{*}=\varphi_{m}$ and $\psi_{n}^{*}=\psi_{n}$. In this way, one can show that if $\lambda$ is an eigenvalue of $K_{1}\left(x_{1}, y_{1}\right)$ and $\mu$ is an eigenvalue of $K_{2}\left(x_{2}, y_{2}\right)$, then $\lambda \mu$ is an eigenvalue of $K(\mathbf{x}, \mathbf{y})$.

Suppose the eigenvalues of $K_{i}\left(x_{i}, y_{i}\right), i=1,2$ are in the order $\mathcal{O}(1), \mathcal{O}\left(R^{-1}\right), \mathcal{O}\left(R^{-2}\right), \ldots$, for some $R>1$, then the eigenvalues of $K(\mathbf{x}, \mathbf{y})$ are expected in the following order: 1 in order $\mathcal{O}(1), 2$ in order $\mathcal{O}\left(R^{-1}\right), 3$ in order $\mathcal{O}\left(R^{-2}\right)$ and $\left(\begin{array}{c}m+1 \\ 1\end{array}\right)$ in order $\mathcal{O}\left(R^{-m}\right)$. See Table 1 for illustration. Such a case can happen, for example, with a separable kernel on a square with an equally spaced $N \times N$ regular mesh. If $K_{1}\left(x_{1}, y_{1}\right)$ is the kernel in the horizontal direction, and $K_{2}\left(x_{2}, y_{2}\right)$ is the kernel in the vertical direction, then their corresponding discrete kernel matrices should have the same eigenvalues because their relative distances in each direction are identical. Similarly, one can show in $\mathbb{R}^{d}$ that, on a $d \times d$ cube with a regular equally spaced mesh, the discrete kernel matrix of a separable kernel are supposed to have $\left(\begin{array}{c}k+d \\ d\end{array}\right)=\frac{(k+d) !}{k !(d) !}$ eigenvalues in similar order. This number is equal to the number of terms in the expansions of

$$
\left(x_{1}+x_{2}+\ldots+x_{d}\right)^{k}
$$

and will become more clear as our discussion proceeds. We first use the analytic and numerical results on Gaussian radial basis functions to verify the result.

Example 1. Consider a weighted inner product defined by $(u(x), v(x))=\int u(x) v(x) w(x) d x$, where $w(x)>0$ is a density function, then the eigenvalues of a kernel are defined by

$$
\int K(x, y) w(y) \psi(y) d y=\lambda \psi(x)
$$




\begin{tabular}{l|ccccccc} 
& 1 & $R^{-1}$ & $R^{-2}$ & $R^{-3}$ & $R^{-4}$ & $R^{-5}$ & $\ldots$ \\
\hline 1 & 1 & $R^{-1}$ & $R^{-2}$ & $R^{-3}$ & $R^{-4}$ & $R^{-5}$ & $\ldots$ \\
$R^{-1}$ & $R^{-1}$ & $R^{-2}$ & $R^{-3}$ & $R^{-4}$ & $R^{-5}$ & $R^{-6}$ &.$\cdot$ \\
$R^{-2}$ & $R^{-2}$ & $R^{-3}$ & $R^{-4}$ & $R^{-5}$ & $R^{-6}$ &.$\cdot$ &.$\cdot$ \\
$R^{-3}$ & $R^{-3}$ & $R^{-4}$ & $R^{-5}$ & $R^{-6}$ &.$\cdot$ &.$\cdot$ &.$\cdot$ \\
$R^{-4}$ & $R^{-4}$ & $R^{-5}$ & $R^{-6}$ &.$\cdot$ &.$\cdot$ &.$\cdot$ &.$\cdot$ \\
$R^{-5}$ & $R^{-5}$ & $R^{-6}$ &.$\cdot$ &.$\cdot$ &.$\cdot$ &.$\cdot$ &. \\
$\vdots$ &.$\cdot$ &.$\cdot$ &.$\cdot$ &.$\cdot$ &.$\cdot$ &.$\cdot$ &.
\end{tabular}

Table 1: The order of eigenvalues of tensor product Kernel in $R^{2}$

With the weighted function $w(x)=\frac{\alpha}{\sqrt{\pi}} e^{-\alpha^{2} x^{2}}, \alpha>0$, the analytic eigenvalues of the Gaussian kernel $e^{-\varepsilon^{2}(x-y)^{2}}$ in $\mathbb{R}$ are given for $n=1,2,3, \cdots$ by

$$
\lambda_{n}=\sqrt{\frac{\alpha^{2}}{\alpha^{2}+\delta^{2}+\varepsilon^{2}}}\left(\frac{1}{1+\delta^{2} / \varepsilon^{2}+\alpha^{2} / \varepsilon^{2}}\right)^{n-1}, \text { where } \delta^{2}=\frac{\alpha^{2}}{2}\left(\sqrt{1+\left(\frac{2 \varepsilon}{\alpha}\right)^{2}}-1\right) .
$$

See[49][30, p.97] for details. Clearly, $1+\delta^{2} / \varepsilon^{2}+\alpha^{2} / \varepsilon^{2}>1$ is the parameter $\rho$ in theorem 6 . For the multivariate Gaussian kernel $K(\mathbf{x}, \mathbf{y})=e^{-\varepsilon_{1}^{2}\left(x_{1}-y_{1}\right)^{2}-\cdots-\varepsilon_{d}^{2}\left(x_{d}-y_{d}\right)^{2}} ;$ we only consider the case $\varepsilon_{1}=\cdots \varepsilon_{d}=\varepsilon$, according to formula (3.6a) in [10, p.A742], the eigenvalues of multivariate Gaussian radial basis function under the weighted inner product with the above weight function can be written as

$$
\lambda_{\mathbf{n}}=\prod_{j=1}^{d} \lambda_{n_{j}}=\left(\frac{\alpha^{2}}{\alpha^{2}+\delta^{2}+\varepsilon^{2}}\right)^{d}\left(\frac{1}{1+\delta^{2} / \varepsilon^{2}+\alpha^{2} / \varepsilon^{2}}\right)^{\sum_{j=1}^{d} n_{j}-d} .
$$

Then if $\sum_{j=1}^{d} n_{j}=k$, then there are $\left(\begin{array}{c}k+d \\ d\end{array}\right)=\frac{(k+d) !}{k ! d !}$ possible combinations of $n_{1}, \cdots, n_{d}$.

\subsection{Infinite Smooth Kernels in $\mathbb{R}^{d}$ With Flat Limit}

The discussion above is primarily based on the properties of the continuous integral operator and pays little attention to the location of the underlying interpolation points. This section concentrates on the discrete form, in particular, on those cases where the shape 


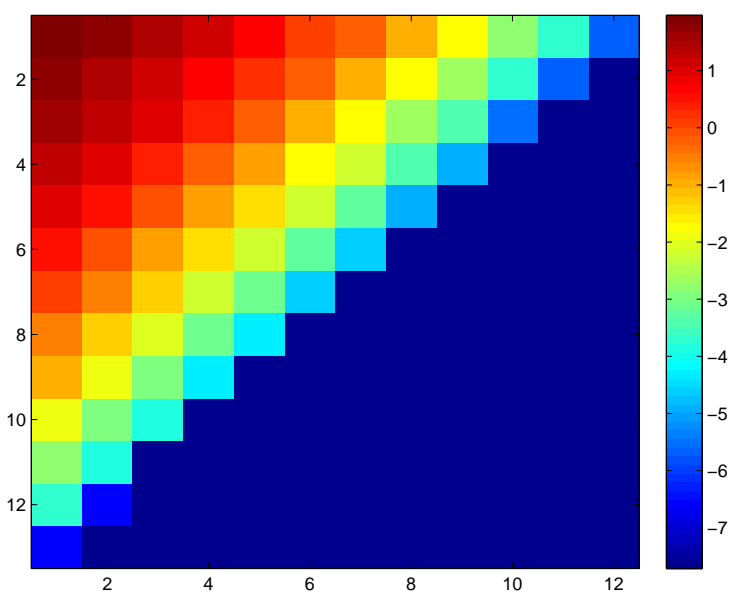

(a) a $9 \times 9$ mesh on unit square

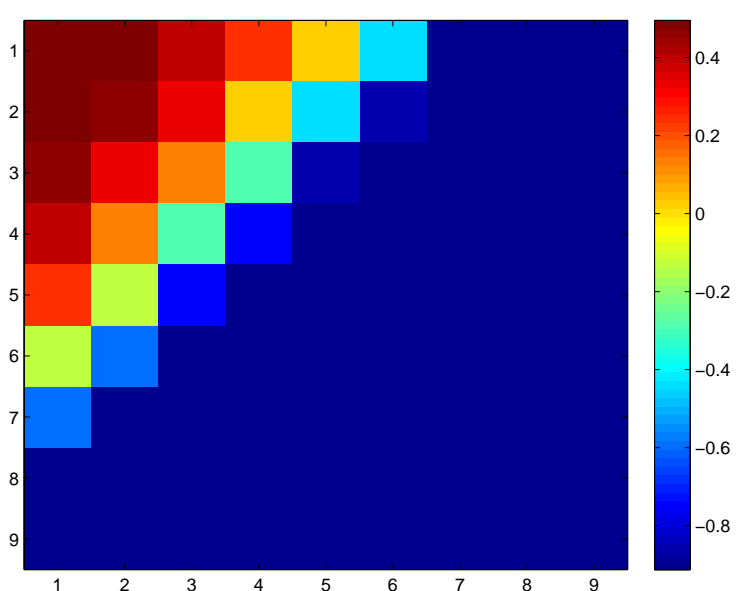

(b) 27 equal spaced points on a circle

Figure 1: Eigenvalues of kernel matrices of Gaussian radial basis function on different meshes. The shape parameter $\varepsilon=5$. We first sort the eigenvalues in descend order, then map the 1 dimensional array to an 2 dimensional diagram by the inverting Cantor pairing function (http://mathworld.wolfram.com/PairingFunction.html). The figure shows the log scale of the eigenvalues by Matlab function imagesc. The dark blue area in the bottom-right corners is empty with NaN. Panel 1(a) demonstrates that, on the regular mesh, the eigenvalues in the same order are grouped by $1,2,3,4, \ldots ;$ while panel 1 (b) shows the eigenvalues are grouped by $1,2,2,2, \ldots$.

parameter of the underlying radial basis function tends to 0 . These radial basis functions with shape parameter $\varepsilon \rightarrow 0$ are called radial basis function with flat limit, and have received a lot attention in recent years [12-14]. The flatness brings the advantage of asymptotic techniques in $\varepsilon$.

Another technique used to investigate the spectral distribution of high dimensional kernels with flatness limit is due to Schaback [43, Theorem 6, p.307]; it doesn't require the kernel function to be separable. In [43], the author focused on a geometric property of the interpolation points and has not concerned explicit results on the distribution of eigenvalues. The basic idea is to construct a sequence of nested subspaces, and investigate quadratic forms corresponding to the interpolation matrix (4). We combine these techniques with other linear algebra results to show the spectral distribution of scaled infinitely smooth radial functions. To prove the results, we introduce the following notation and lemma.

Let $\left\{p_{1}, p_{2}, \ldots, p_{Q}\right\}$ be a basis set for $\pi_{k-1}\left(\mathbb{R}^{d}\right)$, the multivariate polynomial space of degree at most $k-1$, and denote by $\boldsymbol{P}_{k}$ the matrix with entries $\left(p_{j}\left(\mathbf{x}_{i}\right)\right)_{1 \leq i \leq N, 1 \leq j \leq Q}$, then 
for any given data set $\mathcal{X}=\left\{\mathbf{x}_{1}, \mathbf{x}_{2}, \ldots, \mathbf{x}_{N}\right\} \subset \Omega \subset \mathbb{R}^{d}$, we have $\operatorname{ker}\left(\boldsymbol{P}_{k+1}^{T}\right) \subseteq \operatorname{ker}\left(\boldsymbol{P}_{k}^{T}\right)$ for $k=1,2,3, \ldots$ Further denote $\operatorname{ker}\left(\boldsymbol{P}_{0}^{T}\right)=\mathbb{R}^{N}$, then for any finite set $\mathcal{X}$, there is a positive integer $\mu(\mathcal{X})$ such that

$$
\emptyset=\operatorname{ker}\left(\boldsymbol{P}_{\mu(\mathcal{X})}^{T}\right) \subseteq \cdots \subseteq \operatorname{ker}\left(\boldsymbol{P}_{k+1}^{T}\right) \subseteq \operatorname{ker}\left(\boldsymbol{P}_{k}^{T}\right) \subseteq \cdots \subseteq \operatorname{ker}\left(\boldsymbol{P}_{1}^{T}\right) \subset \operatorname{ker}\left(\boldsymbol{P}_{0}^{T}\right)
$$

$\mu(\mathcal{X})$ can be viewed as a geometric property of the data set $\mathcal{X}$, see [43] for details.

Let $\Delta$ be the Eucidean distance matrix with entries $\left(\left\|\mathbf{x}_{i}-\mathbf{x}_{j}\right\|\right)_{1 \leq i, j \leq N}$ and $\Delta^{k}=\left(\| \mathbf{x}_{i}-\right.$ $\left.\mathbf{x}_{j} \|^{k}\right)_{1 \leq i, j \leq N}$ denote the element-wise power of $\boldsymbol{\Delta}$, then there is a well known result due to Micchelli.

Lemma 5 (Micchelli [25]). If $\sum_{i=1}^{n} \alpha_{i} p\left(\mathbf{x}_{i}\right)=0$ for all $p \in \pi_{k-1}\left(\mathbb{R}^{d}\right)$, then the following quadratic form satisfies $(-1)^{k} \alpha^{T} \boldsymbol{\Delta}^{2 k} \alpha \geq 0$, where equality holds if and only if $\sum_{i=1}^{n} \alpha_{i} p\left(x_{i}\right)=$ 0 , for all $p \in \pi_{k}\left(\mathbb{R}^{d}\right)$.

For convenience, we denote the interpolation matrix (4) corresponding to the scaled radial basis function $\phi(\varepsilon r)=g\left(\varepsilon^{2} r^{2}\right)$ by $A_{\phi_{\varepsilon}, \mathcal{X}}=\phi(\varepsilon \boldsymbol{\Delta})=g\left(\varepsilon^{2} \boldsymbol{\Delta}^{2}\right)$. The function $g$ is taken to be infinitely smooth and has a Taylor expansion at the origin.

Theorem 8. Let $\boldsymbol{A}_{\phi_{\varepsilon}, \mathcal{X}}$ be an interpolation matrix corresponding to an infinitely smooth radial basis function $\phi_{\varepsilon}(r)=\phi(\varepsilon r)=g\left((\varepsilon r)^{2}\right)$. If $g$ has an convergent Taylor expansion near the origin in the real line, then, when $\varepsilon \rightarrow 0$, there are exactly $\operatorname{dim}\left(\operatorname{ker}\left(\boldsymbol{P}_{k}^{T}\right)\right)-$ $\operatorname{dim}\left(\operatorname{ker}\left(\boldsymbol{P}_{k+1}^{T}\right)\right)$ eigenvalues behaving like $\varepsilon^{2 k}$, for $0 \leq k \leq \mu(\mathcal{X})-1$, where $\boldsymbol{P}_{k}^{T}$ and $\mu(\mathcal{X})$ are defined in (31). Furthermore, if each $\boldsymbol{P}_{k}$ is full rank, then there are exactly $\left(\begin{array}{c}k+d-1 \\ d-1\end{array}\right)=\frac{(k+d-1) !}{k !(d-1) !}$ eigenvalues behaving like $\varepsilon^{2 k}$.

Proof. Since the function $g$ has a convergent Taylor expansion near the the origin, then we can write the entries of the interpolation matrix as a sum of the element powers of the distance matrix $\Delta$,

$$
\boldsymbol{A}_{\phi_{\varepsilon}, \mathcal{X}}=g\left((\varepsilon \boldsymbol{\Delta})^{2}\right)=\sum_{\ell=0}^{\infty} \frac{g^{(\ell)}(0)}{\ell !}(\varepsilon \boldsymbol{\Delta})^{2 \ell}
$$


Let $m=\mu(\mathcal{X})-1, \boldsymbol{\alpha} \in \operatorname{ker}\left(\boldsymbol{P}_{m}^{T}\right)$, then by Lemma $5 \boldsymbol{\alpha}^{T} \boldsymbol{\Delta}^{2 \ell} \boldsymbol{\alpha}=0$, for $\ell \leq m$. Therefore we have the following quadratic form

$$
\boldsymbol{\alpha}^{T} \boldsymbol{A}_{\phi_{\varepsilon}, \mathcal{X}} \boldsymbol{\alpha}=\varepsilon^{2 m} \frac{f^{(m)}(0)}{m !} \boldsymbol{\alpha}^{T} \boldsymbol{\Delta}^{2 m} \boldsymbol{\alpha}+\sum_{\ell=\mu(X)}^{\infty} \varepsilon^{2 \ell} \frac{f^{(\ell)}(0)}{\ell !} \boldsymbol{\alpha}^{T} \boldsymbol{\Delta}^{2 \ell} \boldsymbol{\alpha}
$$

decays like $\varepsilon^{2 m}$ as $\varepsilon \rightarrow 0$. According the Courant-Fischer's minimum-maximum principle [19, p.179], $A_{\phi_{\varepsilon}, \mathcal{X}}$ has at least $\operatorname{dim}\left(\operatorname{ker}\left(\boldsymbol{P}_{m}^{T}\right)\right)$ eigenvalues which decay at least as fast as $\varepsilon^{2 m}$. Further denote the space $\mathcal{M}_{k} \subseteq \operatorname{ker}\left(\boldsymbol{P}_{k}^{T}\right)$ and $\mathcal{M}_{k} \perp \operatorname{ker}\left(\boldsymbol{P}_{k+1}^{T}\right)$, then

$$
\operatorname{dim}\left(\mathcal{M}_{k}\right)=\operatorname{dim}\left(\operatorname{ker}\left(\boldsymbol{P}_{k}^{T}\right)\right)-\operatorname{dim}\left(\operatorname{ker}\left(\boldsymbol{P}_{k+1}^{T}\right)\right)
$$

Applying the same argument on the space $\mathcal{M}_{k}$, then we can find there are at least $\operatorname{dim}\left(\mathcal{M}_{k}\right)$ eigenvalues decaying at least as $\varepsilon^{2 k}$ in the subspace $\mathcal{M}_{k}$. Since $\sum_{k=1}^{m} \operatorname{dim}\left(\mathcal{M}_{k}\right)=N$, thus there are exactly $\operatorname{dim}\left(\mathcal{M}_{k}\right)$ eigenvalues decaying like $\varepsilon^{2 k}$ when $\varepsilon \rightarrow 0$.

If every $\boldsymbol{P}_{k}$ is full rank, then $\operatorname{rank}\left(\boldsymbol{P}_{k}\right)=\operatorname{dim}\left(\pi_{k-1}\left(\mathbb{R}^{d}\right)\right)=\left(\begin{array}{c}k-1+d \\ d\end{array}\right)$, and

$$
\operatorname{dim}\left(\mathcal{M}_{k}\right)=\operatorname{dim}\left(\pi_{k}\left(\mathbb{R}^{d}\right)\right)-\operatorname{dim}\left(\pi_{k-1}\left(\mathbb{R}^{d}\right)\right)=\frac{(k+d-1) !}{k !(d-1) !}
$$

Note that the number $\left(\begin{array}{c}k-1+d \\ d\end{array}\right)$ is the number of terms in the expansion of $\left(x_{1}+\cdots+x_{d}\right)^{k-1}$, which is consistent with the discussion above, whereas the term $\operatorname{dim}\left(\pi_{k}\left(\mathbb{R}^{d}\right)\right)-\operatorname{dim}\left(\pi_{k-1}\left(\mathbb{R}^{d}\right)\right)$ depends on the geometric property of the interpolation data points. The geometric property is not easy to identify in general. For example, when the interpolation points in a circle with radial 1 , then $\operatorname{dim}\left(\pi_{k}\left(\mathbb{R}^{2}\right)\right)-\operatorname{dim}\left(\pi_{k-1}\left(\mathbb{R}^{2}\right)\right)=2$ for $k=1,2,3$, because $\{1, x, y\}$ are independent, while for $\left\{x^{2}, x y, y^{2}\right\}$, we have $y^{2}=1-x^{2}$, for $\left\{x^{3}, y^{3}, x^{2} y, y^{2} x\right\}$, we have $x^{2} y=y-y^{3}, x y^{2}=x-x^{2}$. It was observed that when the eigenvalues kernel matrices of Gaussian radial basis function on a circle are grouped in different orders, the numbers of each group in descending order are $1,2,2,2,2, \cdots$. The reader is directed to [15, p.389] for more scenarios. Figure 1(b) illustrates the eigenvalues of a kernel matrix of Gaussian radial 
basis function on a circle.

\section{Discussion}

It is noted that there are existing results on the conditioning issue of kernel matrices related to radial basis function. As mentioned, they mainly focused on the smallest eigenvalues and on the condition number. By contrast, here we give simple methods to estimate every eigenvalue, or the distribution of the eigenvalues.

\subsection{Comparison with other results}

Noteworthy results on lower bounds for the smallest eigenvalue include $[1,3,26,29,41$, 42]. The technique employed to prove these results can be summarized as using Fourier transform techniques to estimate a quadratic form in a subspace. There is also one paper which discusses an upper bound on the smallest eigenvalues of the kernel matrices [2], in which the authors construct an special vector, and then use the vector to estimated the inverse norm of the interpolation matrix via some sophisticated results on divided difference formula. Here, we use quite a different way to prove the upper bound of every eigenvalue of the continuous operator. This technique is based on the the Weyl-Courant minimax principle via approximating an infinite dimensional square integral operator by finite Fourier or Chebyshev truncations. After finding the connections between the integral equations and the interpolation problem, the main results are quickly derived. This method can be used in high dimensional space by employing multivariate Fourier approximation.

We mention that the results of Theorem 5 are consistent with previous results. It is likely we get more accurate estimates on the smallest eigenvalue of the kernel matrix. For example for the compactly supported Wendland function, $\phi_{d, k} \in C^{2 k}$, the lower bound of the smallest eigenvalues is $\lambda_{\min } \geq C q^{2 k+1}[47, \mathrm{p} .214]$ for some constant $C$, where the $q$ is the so-called separation distance. For equal space sampling in the interval $[-1,1]$ with the sampling points arranged as $-1=x_{0}<x_{1}<\cdots<x_{n}=1, q=\frac{1}{2} \min _{i=j}\left|x_{i}-x_{j}\right|=\frac{1}{n}$, so that $\lambda_{\min } \geq C n^{-2 k-1}$ . The proof of Theorem 5 shows that $\lambda_{\min }=o\left(n^{-2 k}\right)$. Therefore, we obtain both a lower and upper bound on the smallest eigenvalue for large $n$, say, $C_{1} n^{-2 k-1} \leq \lambda_{\min } \leq C_{2} n^{-2 k}$. 


\subsection{Connection with $Q R-R B F$}

As seen, especially in Theorem 3, the eigenvalues of a kernel have a close relationship with coefficients of the orthogonal expansions of the kernel functions. The eigenvalues of analytic kernels are in the same order as Chebyshev coefficients in the Chebyshev truncations. The recent $\mathrm{RBF}-\mathrm{QR}$ method which aims to compute Gaussian radial basis function interpolants with flat limit is also based on orthogonal expansions [12]. In this method there arises a diagonal scaling matrix with entires which are a part of the coefficients in the orthogonal expansion. Recalling that the eigenvalues of integral operators with periodic kernel are closely related to the Fourier coefficients, for multivariate cases, one might expect, the number of the eigenvalues in the same order is the same as the number of the coefficients of orthogonal expansions with the same (polynomial) order. The number of entries in the order $\varepsilon^{2 k}$ in the scaling matrix in the RBF-QR methods happens to be $\operatorname{dim}\left(\pi_{k}\left(\mathbb{R}^{d}\right)\right)-$ $\operatorname{dim}\left(\pi_{k-1}\left(\mathbb{R}^{d}\right)\right)$, which is the number of the eigenvalues in the corresponding order. This is unlikely to be a coincidence.

The spectral distribution can supply information on how to choose the right diagonal scaling matrix in the RBF-QR method. It can also be used to investigate the smoothing effects of kernel matrices [40] and is closely related to several other computing issues which go beyond discussion in this paper.

\section{Appendix}

Hints of proof of theorem 3

Consider

$$
\lambda u(x)=\int_{-\pi}^{\pi} K(x-y) u(y) d y
$$

where $K(x)$ is integrable and periodic of $2 \pi$. Let

$$
K(x) \sim \sum_{-\infty}^{\infty} \hat{K}_{n} e^{i n x}, \text { where } \hat{K}_{n}=\frac{1}{2 \pi} \int_{-\pi}^{\pi} K(x) e^{-i n x} d x
$$




$$
u(x) \sim \sum_{-\infty}^{\infty} \hat{u}_{n} e^{i n x} \text {, where } \hat{u}_{n}=\frac{1}{2 \pi} \int_{-\pi}^{\pi} u(x) e^{-i n x} d x .
$$

Multiply $e^{-i n x}$ on both side of equation (34), we have

$$
\begin{aligned}
\lambda \hat{u}_{n} & =\frac{1}{2 \pi} \int_{-\pi}^{\pi} u(x) e^{-i n x} d x=\frac{1}{2 \pi} \int_{-\pi}^{\pi} \int_{-\pi}^{\pi} K(x-y) e^{-i n(x-y)} e^{-i n y} u(y) d y d x \\
& =2 \pi \hat{K}_{n} \hat{u}_{n} .
\end{aligned}
$$

\section{Reference}

[1] K. Ball. Eigenvalues of Euclidean distance matrices. J. Approx. Theory, 68(1):74-82, 1992.

[2] K. Ball, N. Sivakumar, and J. D. Ward. On the sensitivity of radial basis interpolation to minimal data separation distance. Constr. Approx., 8(4):401-426, 1992.

[3] B. J. C. Baxter. Norm estimates for inverses of Toeplitz distance matrices. J. Approx. Theory, 79(2):222-242, 1994.

[4] S. Bernstein. Sur la valeur les recherches récentes relatives à la meilleure approximation des fonctions continues par des polynômes. In Proc. 5th. Intern. Math. Congress, v. 1, volume 1, pages 256-266, 1912.

[5] J. Buescu and A.C. Paixão. Eigenvalue distribution of positive definite kernels on unbounded domains. Integral Equations Oper. Theory, 57(1):19-41, 2007.

[6] J. Buescu and A. C. Paixão. Eigenvalue distribution of Mercer-like kernels. Math. Nachr., 280(910):984-995, 2007.

[7] C. H. Chang and C. W. Ha. On eigenvalues of differentiable positive definite kernels. Integral Equations Operator Theory, 33(1):1-7, 1999.

[8] J. A. Cochran. The analysis of linear integral equations. McGraw-Hill Book Co., New York, 1972. McGraw-Hill Series in Modern Applied Mathematics.

[9] F. R. de Hoog. Review of Fredholm equations of the first kind. In Application and numerical solution of integral equations (Proc. Sem., Australian Nat. Univ., Canberra, 1978), volume 6 of Monographs Textbooks Mech. Solids Fluids: Mech. Anal., pages 119-134. Sijthoff \& Noordhoff, Alphen aan den Rijn, 1980.

[10] G. E. Fasshauer and M. J. McCourt. Stable evaluation of Gaussian radial basis function interpolants. SIAM J. Sci. Comput., 34(2):A737-A762, 2012.

[11] J. C. Ferreira and V. A. Menegatto. Eigenvalues of integral operators defined by smooth positive definite kernels. Integral Equations Operator Theory, 64(1):61-81, 2009.

[12] B. Fornberg, E. Larsson, and N. Flyer. Stable computations with Gaussian radial basis functions. SIAM J. Sci. Comput., 33(2):869-892, 2011.

[13] B. Fornberg and C. Piret. A stable algorithm for flat radial basis functions on a sphere. SIAM J. Sci. Comput., 30(1):60-80, 2007/08.

[14] B. Fornberg, G. Wright, and E. Larsson. Some observations regarding interpolants in the limit of flat radial basis functions. Comput. Math. Appl., 47(1):37-55, 2004.

[15] B. Fornberg and J. Zuev. The Runge phenomenon and spatially variable shape parameters in RBF interpolation. Comput. Math. Appl., 54(3):379-398, 2007.

[16] I. Fredholm. Sur une classe d'équations fonctionnelles. Acta Math., 27(1):365-390, 1903. 
[17] C. W. Ha. Eigenvalues of differentiable positive definite kernels. SIAM J. Math. Anal., 17(2):415-419, 1986.

[18] E. Hille and J. D. Tamarkin. On the characteristic values of linear integral equations. Acta Math., $57(1): 1-76,1931$.

[19] R. A. Horn and C. R. Johnson. Matrix analysis. Cambridge University Press, Cambridge, 1990. Corrected reprint of the 1985 original.

[20] Y. Katznelson. An introduction to harmonic analysis. John Wiley \& Sons Inc., New York, 1968.

[21] R. Kress. Linear integral equations, volume 82 of Applied Mathematical Sciences. Springer-Verlag, New York, second edition, 1999.

[22] E. Larsson and B. Fornberg. Theoretical and computational aspects of multivariate interpolation with increasingly flat radial basis functions. Comput. Math. Appl., 49(1):103-130, 2005.

[23] E. Larsson, E. Lehto, E. Heryudono, and B. Fornberg. Stable computation of differentiation matrices and scattered node stencils based on Gaussian radial basis functions. Technical Report 2012-020, Department of Information Technology, Uppsala University, aug 2012.

[24] G. Little and J. B. Reade. Eigenvalues of analytic kernels. SIAM J. Math. Anal., 15(1):133-136, 1984.

[25] C. A. Micchelli. Interpolation of scattered data: distance matrices and conditionally positive definite functions. Constr. Approx., 2(1):11-22, 1986.

[26] F. J. Narcowich and J. D. Ward. Norms of inverses for matrices associated with scattered data. In Curves and surfaces (Chamonix-Mont-Blanc, 1990), pages 341-348. Academic Press, Boston, MA, 1991.

[27] F. J. Narcowich and J.D. Ward. Scattered-data interpolation on $R^{n}$ : error estimates for radial basis and band-limited functions. SIAM J. Math. Anal., 36(1):284-300 (electronic), 2004.

[28] F.J. Narcowich, N. Sivakumar, and J.D. Ward. On condition numbers associated with radial-function interpolation. J. Math. Anal. Appl., 186(2):457-485, 1994.

[29] F.J. Narcowich and J.D. Ward. Norm estimates for the inverses of a general class of scattered-data radial-function interpolation matrices. J. Approx. Theory, 69(1):84-109, 1992.

[30] C. E. Rasmussen and C. K. I. Williams. Gaussian processes for machine learning, volume 1. MIT press Cambridge, MA, 2006.

[31] J. B. Reade. Asymptotic behaviour of eigenvalues of certain integral equations. Proc. Edinburgh Math. Soc. (2), 22(2):137-144, 1979.

[32] J. B. Reade. Eigenvalues of Lipschitz kernels. Math. Proc. Cambridge Philos. Soc., 93(1):135-140, 1983.

[33] J. B. Reade. Eigenvalues of positive definite kernels. SIAM J. Math. Anal., 14(1):152-157, 1983.

[34] J. B. Reade. Eigenvalues of positive definite kernels. II. SIAM J. Math. Anal., 15(1):137-142, 1984.

[35] J. B. Reade. Eigenvalues of smooth kernels. Math. Proc. Cambridge Philos. Soc., 95(1):135-140, 1984.

[36] J. B. Reade. On the sharpness of Weyl's estimate for eigenvalues of smooth kernels. SIAM J. Math. Anal., 16(3):548-550, 1985.

[37] J. B. Reade. Positive definite $C^{p}$ kernels. SIAM J. Math. Anal., 17(2):420-421, 1986.

[38] J. B. Reade. On the sharpness of Weyl's estimates for eigenvalues of smooth kernels. II. SIAM J. Math. Anal., 19(3):627-631, 1988.

[39] J. B. Reade. Eigenvalues of smooth positive definite kernels. Proc. Edinburgh Math. Soc. (2), 35(1):4145, 1992.

[40] R. A. Renaut and S. Zhu. Application of fredholm integral equations inverse theory to the radial basis function approximation problem. Technical Report 1630, The University of Oxford, Nov 2012.

[41] R. Schaback. Lower bounds for norms of inverses of interpolation matrices for radial basis functions. J. Approx. Theory, 79(2):287-306, 1994.

[42] R. Schaback. Error estimates and condition numbers for radial basis function interpolation. Adv. 
Comput. Math., 3(3):251-264, 1995.

[43] R. Schaback. Multivariate interpolation by polynomials and radial basis functions. Constr. Approx., 21(3):293-317, 2005.

[44] F. Smithies. The eigenvalue and singular values of integral equations. Proc. London Math. Soc., 43:255-279, 1937.

[45] L.N. Trefethen. Approximation Theory and Approximation Practice. Applied Mathematics. Society for Industrial and Applied Mathematics, 2013.

[46] A. J. Wathen. Realistic eigenvalue bounds for the Galerkin mass matrix. IMA J. Numer. Anal., $7(4): 449-457,1987$.

[47] H. Wendland. Scattered data approximation, volume 17 of Cambridge Monographs on Applied and Computational Mathematics. Cambridge University Press, Cambridge, 2005.

[48] H. Weyl. Das asymptotische verteilungsgesetz der eigenwerte linearer partieller differentialgleichungen (mit einer anwendung auf die theorie der hohlraumstrahlung). Mathematische Annalen, 71:441-479, 1912. 10.1007/BF01456804.

[49] H. Zhu, C.K.I. Williams, R.J. Rohwer, and M. Morciniec. Gaussian regression and optimal finite dimensional linear models. In C.M. Bishop, editor, Neural Networks and Machine Learning. SpringerVerlag / Berlin, 1998. 